\title{
Decreased TMEM40 expression is associated with malignant behavior of cutaneous squamous cell carcinoma and inhibits tumor progression
}

\author{
LEI YU $^{1 *}$, JIE LIU ${ }^{2 *}$, TANG-DE ZHANG ${ }^{1}$, XIU-FEN ZHENG ${ }^{3}$, DONG-LAN LUO ${ }^{4}$, \\ WEI-LIANG ZHU ${ }^{5}$, XIAN-WEN QIU ${ }^{1}$ and LIN-LANG GUO ${ }^{6}$
}

\begin{abstract}
${ }^{1}$ Department of Dermatology, Zhujiang Hospital of Southern Medical University, Guangzhou, Guangdong 510282;
${ }^{2}$ Department of Clinical Laboratory, Yantai Yuhuangding Hospital, Yantai, Shandong 264000; ${ }^{3}$ Department of Dermatology, Shunde Hospital of Southern Medical University, Shunde, Guangdong 528308; ${ }^{4}$ Department of Dermatology, Shenzhen Maternity and Child Healthcare Hospital, Southern Medical University, Shenzhen, Guangdong 518028; Departments of ${ }^{5}$ Oncology and ${ }^{6}$ Pathology, Zhujiang Hospital of Southern Medical University, Guangzhou, Guangdong 510282, P.R. China
\end{abstract}

Received June 16, 2020; Accepted March 8, 2021

DOI: $10.3892 / \mathrm{ol} .2021 .12867$

\begin{abstract}
Cutaneous squamous cell carcinoma (CSCC) is one of the most common types of skin cancer in humans worldwide. The identification and characterization of cancer-associated transmembrane proteins are important for understanding the molecular biology of CSCC. The aim of the present study was to evaluate the expression pattern of transmembrane protein 40 (TMEM40) in CSCC and its clinical significance. The underlying mechanisms were also examined. Reverse transcription-quantitative PCR, western blot and immunohistochemistry analysis were used to determine the relative expression of TMEM40 in CSCC cell lines and clinical tissue samples. The effect of TMEM40 gene silencing on cell proliferation was also evaluated using Cell Counting Kit- 8 assays. Wound healing assays, flow cytometry and Transwell assays were used to explore the migration, cell cycle distribution/apoptosis and invasion of CSCC cells following TMEM40 silencing, respectively. In the present study, increased TMEM40 expression was observed in CSCC tissue samples, compared with normal skin, and TMEM40 expression was associated with large tumor size in patients
\end{abstract}

Correspondence to: Dr Lin-Lang Guo, Department of Pathology, Zhujiang Hospital of Southern Medical University, 253 Gongye Avenue, Guangzhou, Guangdong 510282, P.R. China

E-mail: linlangg@yahoo.com

*Contributed equally

Abbreviations: CSCC, cutaneous squamous cell carcinoma; IHC, immunohistochemistry; TMEM40, transmembrane protein 40; CCK-8, Cell Counting Kit-8; NC, negative control; siRNA, small interfering RNA

Key words: TMEM40, CSCC, biomarker, migration, apoptosis with CSCC. In vitro functional assays indicated that TMEM40 was involved in the regulation of A431 and SCL1 cell growth through its effects on the cell cycle and apoptosis. Silencing TMEM40 in A431 and SCL1 cells resulted in cell cycle arrest at the $G_{0} / G_{1}$ phase and promoted apoptosis. In addition, migration and invasion were significantly inhibited following silencing of TMEM40 expression in CSCC cells. Taken together, the results of the present study indicated that reduced TMEM40 expression could inhibit CSCC development and that TMEM40 may represent a therapeutic target in CSCC.

\section{Introduction}

Skin cancer arises from the uncontrolled growth of keratinocytes and primarily develops upon exposure to ultraviolet radiation from tanning beds or sunlight (1). It can be classified as basal cell carcinoma, melanoma or cutaneous squamous cell carcinoma (CSCC) (2). Epidemiological studies indicate that CSCC accounts for $20 \%$ of all skin cancers and is associated with a continuously increasing incidence worldwide, posing a threat to public health (3). CSCC is a common type of malignant skin tumor derived from epidermal malpighian cells (4). Furthermore, the metastasis, invasion and local recurrence of CSCC are still the major reasons for treatment failure in most patients (5).

Transmembrane protein 40 (TMEM40) is a multi-pass membrane protein consisting of 233 amino acids, with two isoforms (6-9). Its coding gene is located on chromosome 3 p25.2 in humans. TMEM40 plays a role in collagen-induced arthritis (10-12). Notably, in bladder cancer and tongue squamous cell carcinoma, TMEM40 expression levels are significantly associated with clinical stage, histological grade, pathological grade and primary tumor (pT) status $(13,14)$. However, the specific function of TMEM40 in CSCC, especially the underlying molecular mechanisms by which TMEM40 performs its functions and modulates the malignant behavior of CSCC cells, requires further study. Several recent 
studies have focused on CSCC, with the aim of reducing mortality rates; however, the overall pathophysiological and molecular mechanisms underlying the role of TMEM40 in CSCC remain largely unknown $(15,16)$.

In the present study, to evaluate the role of TMEM40 in CSCC development, the expression levels of TMEM40 were examined in CSCC tissue compared with matched adjacent normal tissue samples. The potential associations between TMEM40 levels and clinicopathological features were also determined. Furthermore, the potential role of TMEM40 in the proliferation, migration and invasion of CSCC cells was also evaluated in vitro. The results suggested that TMEM40 serves an important role in CSCC cell proliferation and migration and may represent a potential therapeutic target in patients with CSCC.

\section{Materials and methods}

Tissue sample collection. A total of 40 participants (17 females and 23 males) diagnosed with CSCC were included in the present study. The mean $( \pm \mathrm{SD})$ age was $61.9( \pm 11.2)$ years, ranging between 33 and 87 years. The inclusion criteria were: i) Diagnosis as CSCC (17); ii) initial diagnosis and no previous treatment; and iii) availability of detailed medical records and follow-up visit. Exclusion criteria were non-cutaneous SCC, CSCC that had been previously treated, cutaneous carcinoma in situ, unavailability of detailed medical records and follow-up visit. Paraffin-embedded tissues were obtained from patients with CSCC. A total of 40 CSCC tissue samples and 40 matched adjacent normal cutaneous tissue samples $(10 \mathrm{~mm}$ from cancer) were collected from preoperative treatment-naïve patients with CSCC who had undergone surgical intervention between May 2018 and June 2019 at Zhujiang Hospital of Southern Medical University (Guangzhou, China). All tissue samples were obtained with written informed consent from the patients involved in this research project. The present study was approved by the Medical Ethics Committee of Zhujiang Hospital of Southern Medical University (approval no. 2020-KY-059-01).

Tissue microarray (TMA) construction and immunohistochemistry. According to standard methods $(10,14,18)$, a TMA was constructed using 40 CSCC paraffin-embedded tissues and 40 normal cutaneous paraffin-embedded tissue samples. The tissues were fixed with $4 \%$ paraformaldehyde for $12 \mathrm{~h}$ at $4^{\circ} \mathrm{C}$. The slice thickness was $6 \mu \mathrm{m}$ and sections were stained by immunohistochemistry. The sections were deparaffinized in xylene and hydrated using gradient ethanol solutions. The sections were then blocked in PBS containing 5\% normal goat serum (cat. no. 16210072; Gibco; Thermo Fisher Scientific, Inc.) for $10 \mathrm{~min}$ at room temperature and subsequently incubated with TMEM40-specific primary antibody (1:100; cat. no. sc-393601; Santa Cruz Biotechnology, Inc.) overnight at $4^{\circ} \mathrm{C}$. After being washed three times in 1X PBS, the sections were incubated with horseradish peroxidase-conjugated secondary antibody (1:1,000; cat. no. sc-2357; Santa Cruz Biotechnology, Inc.) for $1 \mathrm{~h}$ at room temperature. After additional 1X PBS washing, the sections were visualized using a DAB Horseradish Peroxidase Color Development kit (cat. no. P0202; Beyotime Institute of Biotechnology) and counterstained with hematoxylin for $2 \mathrm{~min}$ at room temperature.

TMEM40 expression was assessed semi-quantitatively by scoring the percentage positive cells and staining intensity by two observers under a light microscope (magnifications, x40 and x100; Nikon Eclipse TiS; Nikon Corporation). The extent of TMEM40 immunoreactivity was scored as: i) 0 , for $<5 \%$ positive cells; ii) 1 , for $5-25 \%$ positive cells; iii) 2 , for $26-50 \%$ positive cells; iv) $3,51-75 \%$ positive cells; and v) 4 , for 76-100\% positive cells. The intensity of TMEM 40 was scored as: i) 0 , no staining; ii) 1 , light yellow; iii) 2 , brown; and iv) 3 , dark brown. The overall staining score was obtained by multiplying the immunoreactivity score by the intensity score to obtain four grades: i) Negative, for an overall score of 0; ii) weakly positive, for an overall score of $1-4$; iii) moderately positive, for an overall score of 5-8; and iv) strongly positive for an overall score of 9-12 (10).

Cell culture. The CSCC cell lines (A431 and SCL-1) were purchased from the China Center for Type Culture Collection. A431 and SCL1 cells were cultured in high-glucose DMEM (Thermo Fisher Scientific, Inc.) supplemented with 10\% FBS (Gibco; Thermo Fisher Scientific, Inc.) and 1\% penicillin/streptomycin (Biowest) in an incubator at $37^{\circ} \mathrm{C}$ with a humidified atmosphere of $5 \% \mathrm{CO}_{2}$ and $95 \%$ air.

TMEM40-small interfering RNA (siRNA) transfection. The siRNA targeting TMEM40 and negative control (NC)-siRNA were supplied by Invitrogen (Thermo Fisher Scientific, Inc.), and the sequence was verified by sequencing. A total of 5 nmol siRNA and NC were transfected into CSCC cells with Lipofectamine ${ }^{\circledR} 2000$ (Invitrogen; Thermo Fisher Scientific, Inc.) for $20 \mathrm{~min}$ at room temperature according to the manufacturer's instructions. The control group was treated with PBS. The transfection efficiency was assessed by flow cytometry (MoFlo XDP; Beckman Coulter, Inc.). Briefly, after $48 \mathrm{~h}$ of transfection with fluorescently labeled with 6-carboxy-fluorescein (FAM) TMEM40-siRNA, $1 \times 10^{5}$ cells were collected and the percentage of FAM-positive cells was obtained using a flow cytometer, representing the transfection efficiency, and was analyzed with FlowJo software (version 7.6; FlowJo LLC). The sequence of TMEM40-siRNA was 5'-GUGGACGCCUCUCAGUUAA-3', and the non-targeting NC-siRNA sequence was 5'-TTCTCC GAACGTGTCACGT-3'.

Western blotting. To detect TMEM40 protein expression, tissue samples (8 paired normal and tumor tissues) were lysed, and proteins were extracted using radioimmunoprecipitation assay buffer containing $1 \mathrm{mM}$ PMSF (Beyotime Institute of Biotechnology) according to standard protocols. The protein concentrations in the supernatants were determined using a BCA Protein Assay kit (cat. no. P0006; Beyotime Institute of Biotechnology). Subsequently, $\sim 40 \mu \mathrm{g}$ of each total protein sample were separated by SDS-PAGE on $10 \%$ gels and transferred to nitrocellulose membranes, which were then blocked with $5 \%$ fat-free milk for $2 \mathrm{~h}$ at room temperature. The membranes were then washed with TBST and incubated overnight at $4{ }^{\circ} \mathrm{C}$ with a mouse anti-TMEM40 primary antibody (1:500; cat. no. sc-393601; Santa Cruz Biotechnology, Inc.) and mouse anti-GAPDH primary antibody $(1: 1,000$; 
Table I. Primer sequences.

\begin{tabular}{lc}
\hline Primer name & \multicolumn{1}{c}{ Primer sequence $\left(5^{\prime}-3^{\prime}\right)$} \\
\hline TMEM40-F & GCGGTAGGGGTGTACGGT \\
TMEM40-R & CCGGACACGCTGAACTTGT \\
GAPDH-F & CAGCCTCAAGATCATCAGCA \\
GAPDH-R & TGTGGTCATGAGTCCTTCCA
\end{tabular}

F, forward; R, reverse; TMEM40, transmembrane protein 40.

cat. no. 60004-1-Ig; ProteinTech Group, Inc.). After washing, membranes were incubated with appropriate HRP-conjugated secondary antibodies (1:5,000; cat. no. sc-2357; Santa Cruz Biotechnology, Inc.) for $1 \mathrm{~h}$ at room temperature. The signals on the membrane were detected using an enhanced chemiluminescent reagent kit (cat. no. 36222ES60; Shanghai Yeasen Biotechnology Co., Ltd.).

RNA isolation and reverse transcription-quantitative PCR (RT-qPCR). To determine TMEM40 mRNA expression levels, total RNA was extracted from clinical samples or from A431 and SCL-1 cells $48 \mathrm{~h}$ after transfection using a PureLink RNA Mini kit (Thermo Fisher Scientific, Inc.) according to the manufacturer's protocol. Total RNA was quantified using a NanoDrop ${ }^{\mathrm{TM}} 2000$ spectrophotometer (Thermo Fisher Scientific, Inc.). A TaqMan MicroRNA Reverse Transcription kit (Applied Biosystems; Thermo Fisher Scientific, Inc.) was used to reverse transcribe cDNA from total RNA according to the manufacturer's instructions. SYBR-Green PCR kit (Takara Biotechnology Co., Ltd.) and an ABI 7500 realtime PCR amplifier (Applied Biosystems; Thermo Fisher Scientific, Inc.) were used for qPCR. The thermocycling conditions were as follows: $95^{\circ} \mathrm{C}$ for $10 \mathrm{~min}$, followed by 40 cycles at $95^{\circ} \mathrm{C}$ for $15 \mathrm{sec}$ and $60^{\circ} \mathrm{C}$ for $34 \mathrm{sec}$. GAPDH was used for normalization. The relative gene expression data were detected by qPCR and analysed with the $2^{-\Delta \Delta C q}$ method (19). The RT-qPCR primers and their sequences are listed in Table I.

Cell proliferation assay. Cell proliferation was measured using Cell Counting Kit-8 (Nanjing KeyGen Biotech Co., Ltd.). Briefly, a total of $1.5 \times 10^{3} \mathrm{~A} 431$ and SCL-1 cells were plated into 96-well microplates (Corning, Inc.). After transfection, cells were incubated with CCK-8 reagent (Beyotime Institute of Biotechnology) for $2 \mathrm{~h}$ at $37^{\circ} \mathrm{C}$, and the absorbance was measured at $450 \mathrm{~nm}$ using a microplate reader (Infinite ${ }^{\circledR}$ M200; Tecan Group, Ltd.) according to the manufacturer's instructions.

Wound healing assay. A wound healing assay was used to assess cell migration. A total of $1 \times 10^{4}$ A431 and SCL- 1 cells were seeded in 6-well plates, transfected for $48 \mathrm{~h}$ and cultured with serum-free medium to $85-90 \%$ confluence. The cell layers were scratched using a $10 \mu \mathrm{l}$ RNase-free pipette tip to create wound gaps. The wound gaps were imaged at two different time points ( 0 and $48 \mathrm{~h}$ ) using an light microscope (magnification, x40) and analyzed by measuring the distance that the cells migrated in three different areas of each wound.
Transwell migration and invasion assays. The migration and invasion (inserts were pre-coated with Matrigel for $4 \mathrm{~h}$ at $37^{\circ} \mathrm{C}$ ) of A431 and SCL-1 cells were examined using Transwell assays with modified 24-well Boyden chambers (8- $\mu \mathrm{m}$ pore size; Corning, Inc.). A total of $3 \times 10^{4}$ cells in $300 \mu \mathrm{l}$ of DMEM were seeded into the upper chamber of the Transwell inserts $24 \mathrm{~h}$ after TMEM40-siRNA transfection, and $700 \mu \mathrm{l}$ of DMEM supplemented with $20 \%$ FBS was added to the lower chambers. Cells were incubated at $37^{\circ} \mathrm{C}$ in $5 \% \mathrm{CO}_{2}$ for $36 \mathrm{~h}$ after plating. The cells were then fixed with $100 \%$ methanol for $5 \mathrm{~min}$ at room temperature and stained with $0.5 \%$ crystal violet at room temperature for $15 \mathrm{~min}$. The numbers of migrating or invading cells that passed through the membrane were counted in three randomly selected fields under a light microscope (magnification, $x 40$ ), and the average value was calculated with ImageJ software (version 1.8; National Institutes of Health).

Cell cycle analysis. A431 and SCL-1 cells were fixed overnight in $70 \%$ ethanol at $-20^{\circ} \mathrm{C}$ after digestion with $0.05 \%$ trypsin for $3 \mathrm{~min}$ at room temperature. (Nanjing KeyGen Biotech Co., Ltd.). The fixed cells were stained with $450 \mu \mathrm{l}$ propidium iodide (PI; Nanjing KeyGen Biotech Co., Ltd.) and $50 \mu$ l RNase A (KeyGen Biotech, Nanjing) at room temperature in the dark for $30 \mathrm{~min}$. Cell cycle progression was examined using a flow cytometer (MoFlo XDP; Beckman Coulter, Inc.) and analyzed using FlowJo software (version 7.6; FlowJo LLC).

Apoptosis assessment. Flow cytometry (MoFlo XDP; Beckman Coulter, Inc.) was used to evaluate the apoptosis rate in transfected cells. SCL1 and A431 cells $\left(6 \times 10^{5}\right)$ were plated in $60-\mathrm{mm}$ dishes and incubated overnight. The cells were then transiently transfected with TMEM40-siRNA or control vector for $48 \mathrm{~h}$. The cells were then collected by trypsinization, washed with cold PBS and stained using a FITC Annexin V/Dead Cell Apoptosis kit (Nanjing KeyGen Biotech Co., Ltd.) at room temperature for $30 \mathrm{~min}$. The apoptosis rates of the CSCC cells were analyzed using FlowJo software (version 7.6; FlowJo LLC).

Statistical analysis. Statistical analysis was performed using the SPSS (version 20.0; IBM Corp.) or GraphPad Prism software (version 5.0; GraphPad Software, Inc.). The differences between the CSCC tissue and paired normal tissue groups were evaluated using a paired Student's t-test. One-way ANOVA followed by Tukey's post hoc test were used for multiple comparisons. Differences between groups were tested using the $\chi^{2}$ or Fisher's exact test $(\mathrm{n}<5)$ test for categorical variables. The results are presented as the mean \pm SD. $\mathrm{P}<0.05$ was considered to indicate a statistically significant difference.

\section{Results}

TMEM40 expression is upregulated in CSCC tissue samples. To investigate the role of TMEM40 in CSCC, the expression of TMEM40 was evaluated in patient samples using immunohistochemistry. TMEM40 expression was significantly upregulated in CSCC tissue compared with adjacent normal skin tissue samples (Fig. 1A-H; Table II). As shown in Table III, TMEM40 protein expression was significantly 
Table II. TMEM40 expression in paired CSCC and normal skin tissue samples $(\mathrm{n}=40)$.

\begin{tabular}{lccr}
\hline Tissue type & $\begin{array}{c}\text { Negative TMEM40 } \\
\text { expression, } \mathrm{n}(\%)\end{array}$ & $\begin{array}{c}\text { Positive TMEM40 } \\
\text { expression, } \mathrm{n}(\%)\end{array}$ & P-value $^{\mathrm{a}}$ \\
\hline CSCC & $29(72.5)$ & $11(27.5)$ & $<0.001$ \\
Normal skin & $9(22.5)$ & $31(77.5)$ & \\
\hline
\end{tabular}

${ }^{a} \chi^{2}$ test. TMEM40, transmembrane protein 40; CSCC, cutaneous squamous cell carcinoma.

A

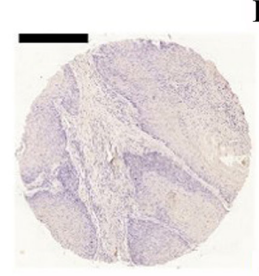

E

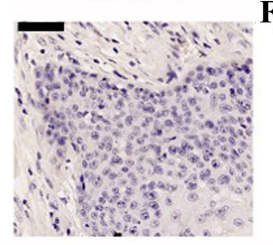

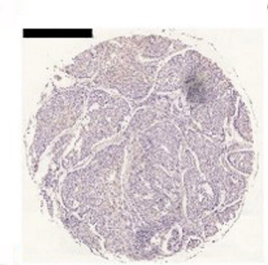

$\mathbf{F}$

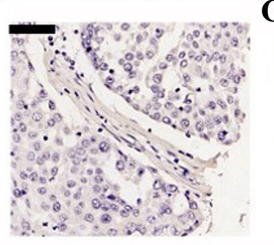

C

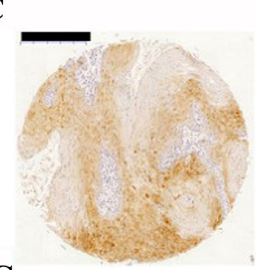

$\mathbf{G}$

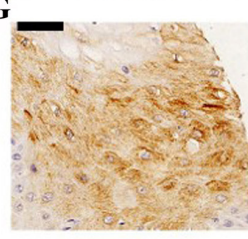

D

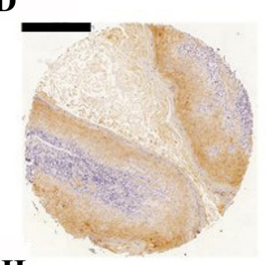

$\mathbf{H}$

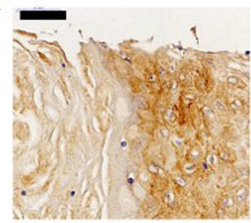

I

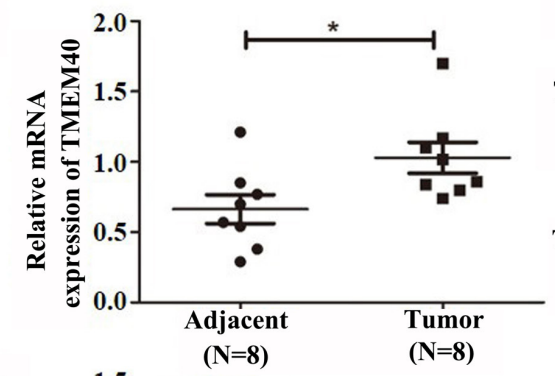

$\mathbf{K}$

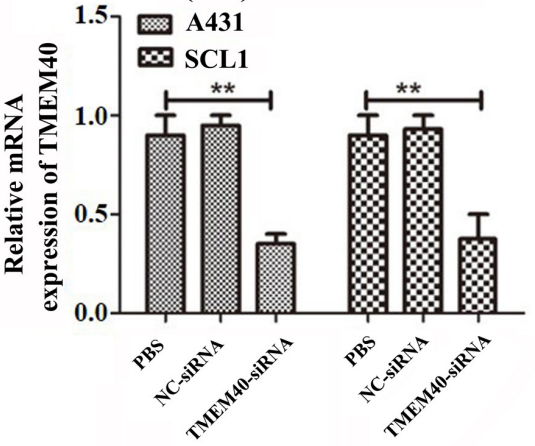

$\mathbf{J}$

$\begin{array}{llllllllllllllll} & \mathrm{N} 1 & \mathrm{~T} 1 & \mathrm{~N} 2 & \mathrm{~T} 2 & \mathrm{~N} 3 & \mathrm{~T} 3 & \mathrm{~N} 4 & \mathrm{~T} 4\end{array}$

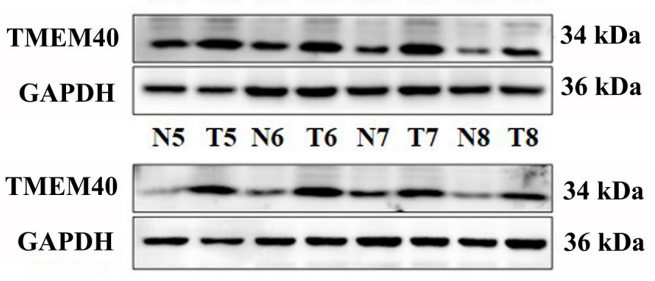

$\mathbf{L}$

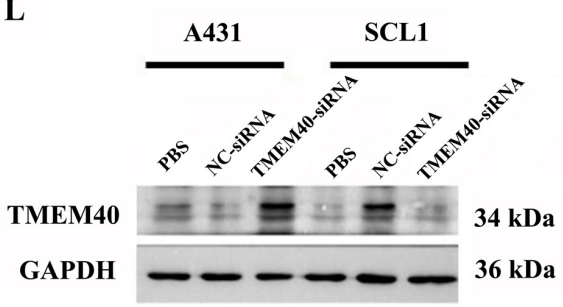

Figure 1. TMEM40 was expressed in CSCC tissues and cell lines. (A-D) Immunohistochemical of TMEM40 protein expression was significantly increased in CSCC compared with normal cutaneous squamous cells. Magnification, x5. Scale bar, 500 $\mu \mathrm{M}$. (E-H) High-magnification immunohistochemical staining of TMEM40. Magnification, x40. Scale bar, $50 \mu \mathrm{m}$. TMEM40 (I) mRNA and (J) protein expression was significantly increased in CSCC compared with normal cervical tissue. $\mathrm{n}=8$ in each group. TMEM40-siRNA significantly downregulates the expression of TMEM40 in A431 and SCL1 cells at the (K) mRNA and (L) protein levels. ${ }^{*} \mathrm{P}<0.05 ;{ }^{* *} \mathrm{P}<0.01$. CSCC, cutaneous squamous cell carcinoma; NC, negative control; $\mathrm{N}$, normal tissue; $\mathrm{T}$, tumor tissue; TMEM40, transmembrane protein 40; siRNA, small interfering RNA.

associated with tumor size $(\mathrm{P}=0.007)$. However, there was no significant association between TMEM40 expression and age, pathological grade, lymph node metastasis or pT status (all $\mathrm{P}>0.05$ ). To determine the expression of TMEM40 in CSCC at the mRNA and protein levels, western blot and RT-qPCR analysis were performed in CSCC tissue and paired adjacent normal skin tissue samples (n=8; Fig. 1I and J). All of these results suggested that TMEM40 expression was upregulated in CSCC tissue compared with normal adjacent tissue.

Downregulation of TMEM40 inhibits CSCC cell proliferation. TMEM40-siRNA transfection was used to silence the 
Table III. Association between TMEM40 expression and clinicopathological features in squamous cell carcinoma tissues.

\begin{tabular}{|c|c|c|c|c|}
\hline \multirow{2}{*}{$\begin{array}{l}\text { Clinicopathological } \\
\text { features }\end{array}$} & \multirow[b]{2}{*}{ Total patients, $\mathrm{n}$} & \multicolumn{2}{|c|}{ TMEM40 protein expression } & \multirow[b]{2}{*}{ P-value } \\
\hline & & Negative, n (\%) & Positive, n (\%) & \\
\hline All patients & 40 & $9(22.5)$ & $31(77.5)$ & Not applicable \\
\hline Age, years & & & & 0.456 \\
\hline$\leq 48$ & 23 & $4(17.4)$ & $19(82.6)$ & \\
\hline$>48$ & 17 & $5(29.4)$ & $12(70.6)$ & \\
\hline Lymph node metastasis & & & & 0.061 \\
\hline No & 24 & $8(33.3)$ & $16(66.7)$ & \\
\hline Yes & 16 & $1(6.3)$ & $15(93.7)$ & \\
\hline Tumor size, $\mathrm{cm}$ & & & & 0.007 \\
\hline$<3$ & 11 & $6(54.5)$ & $5(45.5)$ & \\
\hline$\geq 3$ & 29 & $3(10.3)$ & $26(89.7)$ & \\
\hline Pathological grade & & & & 0.265 \\
\hline I & 19 & $6(31.6)$ & $13(68.4)$ & \\
\hline II-III & 21 & 3 (14.2) & $18(85.8)$ & \\
\hline pT status & & & & 0.120 \\
\hline $\mathrm{T} 1$ & 13 & $5(38.5)$ & $8(61.5)$ & \\
\hline T2-T3 & 27 & $4(14.8)$ & $23(85.2)$ & \\
\hline
\end{tabular}

${ }^{\text {a}}$ Fisher's exact test. pT, primary tumor; TMEM40, transmembrane protein 40.

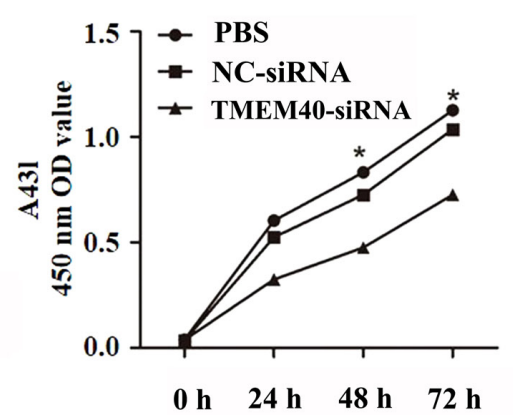

C

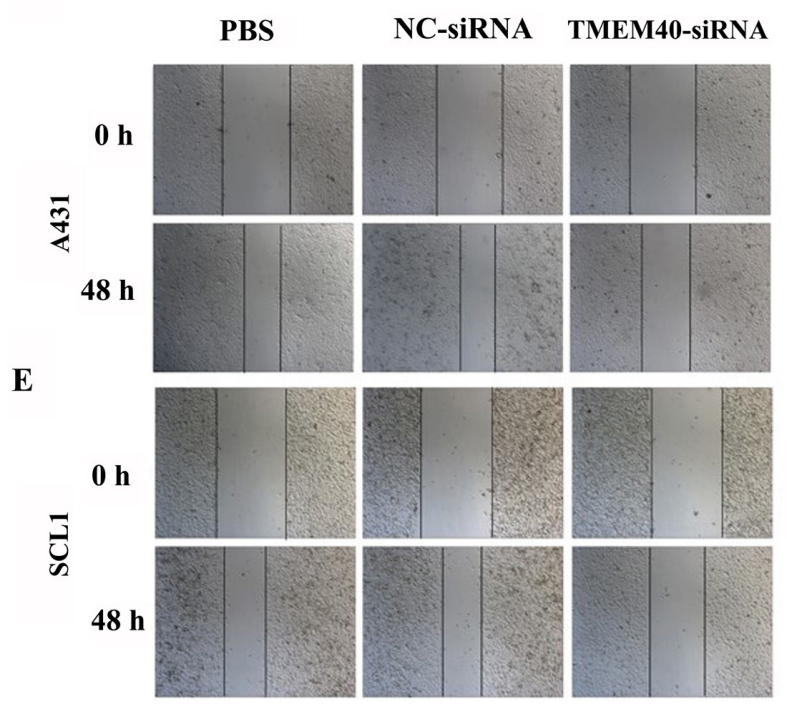

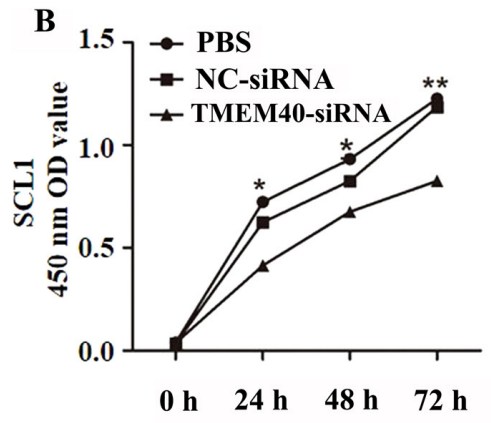

D
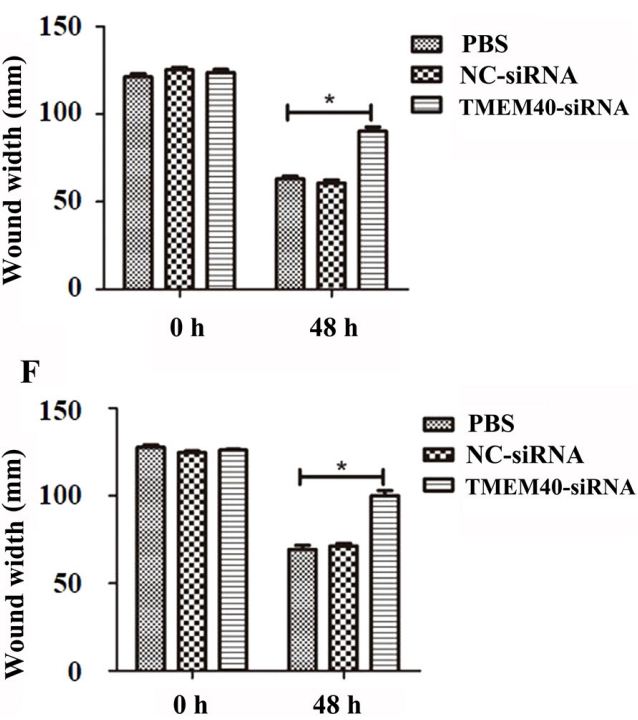

Figure 2. Knockdown of TMEM40 expression suppresses the viability of CSCC cells. Cell proliferation was determined in (A) A431 and (B) SCL1 cells ${ }^{*} \mathrm{P}<0.05$ and ${ }^{* *} \mathrm{P}<0.01$ vs. TMEM40-siRNA. Wound healing assays in (C and D) A431 and (E and F) SCL1 cells following transfection with TMEM40-siRNA and NC-siRNA or PBS treatment. Magnification, $x 40 .{ }^{*} \mathrm{P}<0.05$. CSCC, cutaneous squamous cell carcinoma; NC, negative control; OD, optical density; TMEM40, transmembrane protein 40; siRNA, small interfering RNA. 
A

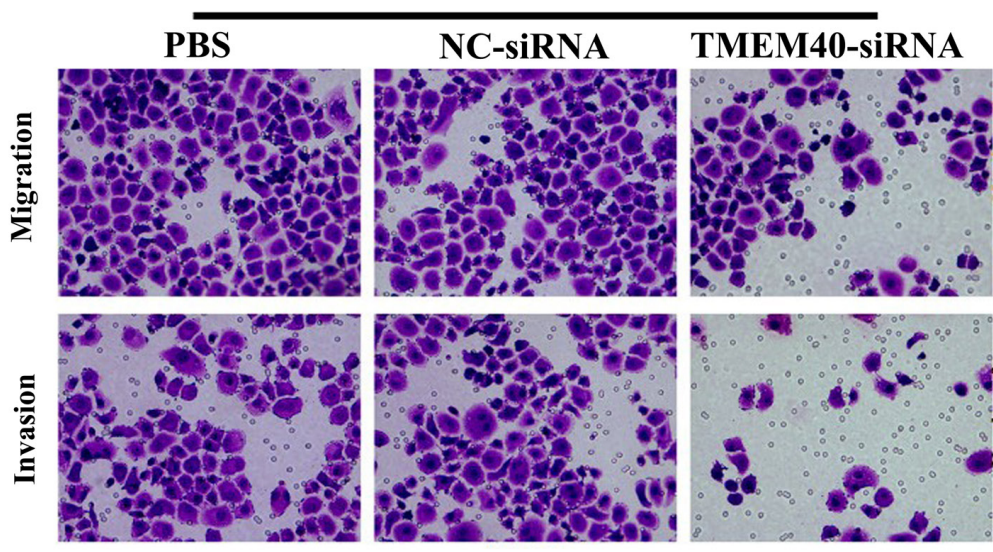

D

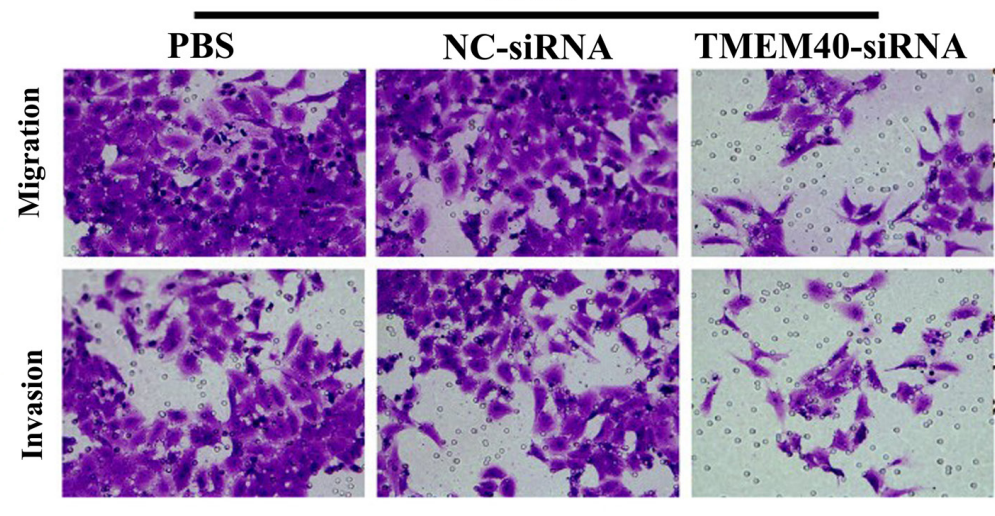

B

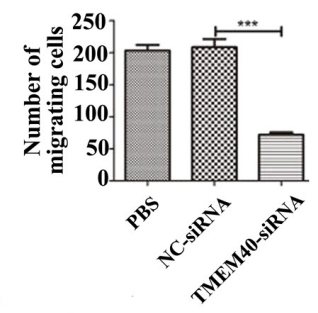

C

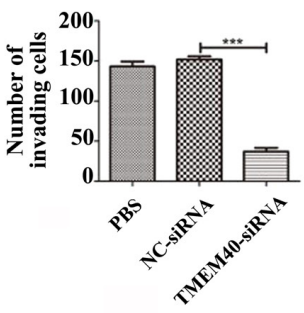

E

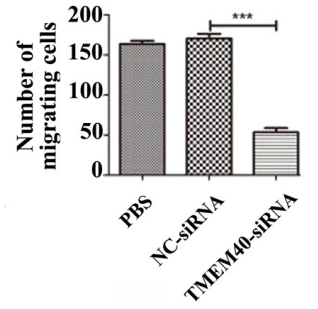

F

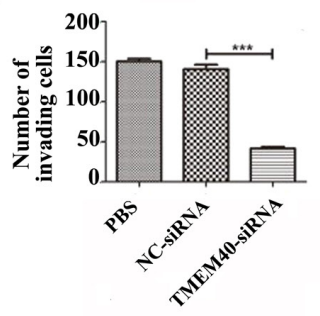

Figure 3. TMEM40 regulates CSCC cell invasion and migration in vitro. (A) Transwell assays were used to measure (B) migration and (C) invasion in A431 cells following transfection with TMEM40-siRNA and NC-siRNA or PBS treatment. (D) Transwell assays were used to measure (E) migration and (F) invasion in SCL1 cells following transfection with TMEM40-siRNA and NC-siRNA or PBS treatment. Magnification, $\mathrm{x} 100$. ${ }^{* * *} \mathrm{P}<0.001$. CSCC, cutaneous squamous cell carcinoma; NC, negative control; TMEM40, transmembrane protein 40; siRNA, small interfering RNA.

expression of the TMEM40 gene in A431 and SCL1 cells. TMEM40-siRNA group exhibited significantly decreased TMEM40 expression compared with the other groups (Fig. 1K and L). TMEM40-siRNA and NC transfection efficiency were 77.9 and $73.8 \%$ in A431 cells, respectively, and 74.1 and $75.7 \%$ in SCL1 cells, respectively (Fig. S1). These transfected cells were used in the following experiments. A CCK-8 assay was performed to study the effect of TMEM40 expression on CSCC cell growth. The growth curves demonstrated that cell proliferation was significantly decreased in the TMEM40-siRNA group compared with in the NC-siRNA and PBS groups in both A431 (at 48 and $72 \mathrm{~h}$ ) and SCL1 cells (at 24, 48 and 72 h) (Fig. 2A and B).

TMEM40 silencing inhibits the migration andinvasion of CSCC cells. Wound healing and Transwell assays were performed to determine whether the TMEM40 gene could affect the migration and invasion of CSCC cells. Wound healing assay shown in Fig. 2C and E suggested that TMEM40-knockdown significantly slowed wound closure. The width of the wound of A431 (Fig. 2D) and SCL1 (Fig. 2F) cells was significantly higher in the TMEM40-silenced groups than in the other groups. Subsequently, cell migration and invasion was also detected with Transwell assays. As shown in Fig. 3, significantly fewer migrating and invading cells were observed in the TMEM40-siRNA group compared with in the other groups.

Silencing TMEM40 is associated with cell cycle regulation and apoptosis. To determine the role of TMEM40 in cell growth, apoptosis and cell cycle distribution were assessed in A431 and SCL1 following TMEM 40 knockdown. The results demonstrated that TMEM 40 silencing induced $\mathrm{G}_{0} / \mathrm{G}_{1}$-phase arrest in $\mathrm{A} 431$ and SCL1 cells (Fig. 4A), and the percentage of cells in $\mathrm{G}_{0} / \mathrm{G}_{1}$ phase in the TMEM40-silenced group was higher than that in the other groups (Fig. 4B and C). Moreover, there was a significantly higher percentage of AnnexinV $\mathrm{PI}^{+}$(late apoptotic) cells and AnnexinV $\mathrm{PI}^{+}$(early apoptotic) cells among TMEM40-silenced CSCC cells than in the other groups (Fig. 4D-F), indicating that TMEM40- silencing significantly increased apoptosis.

\section{Discussion}

CSCC is a major public health concern due to its associated medical costs and high incidence (20). The known predisposing 
A

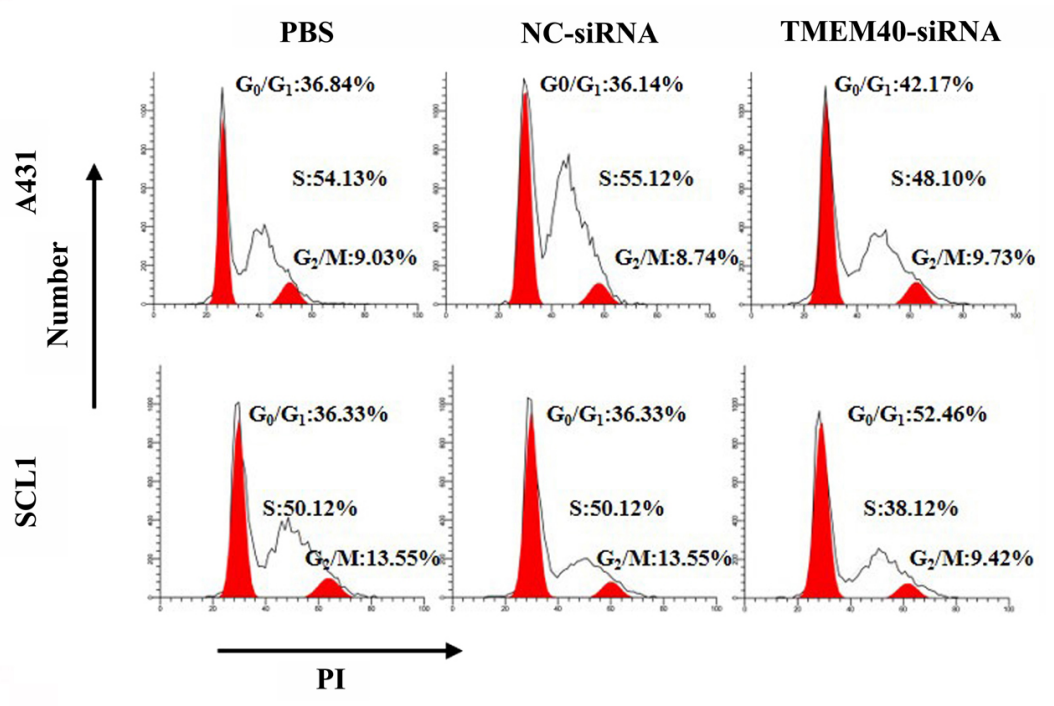

B
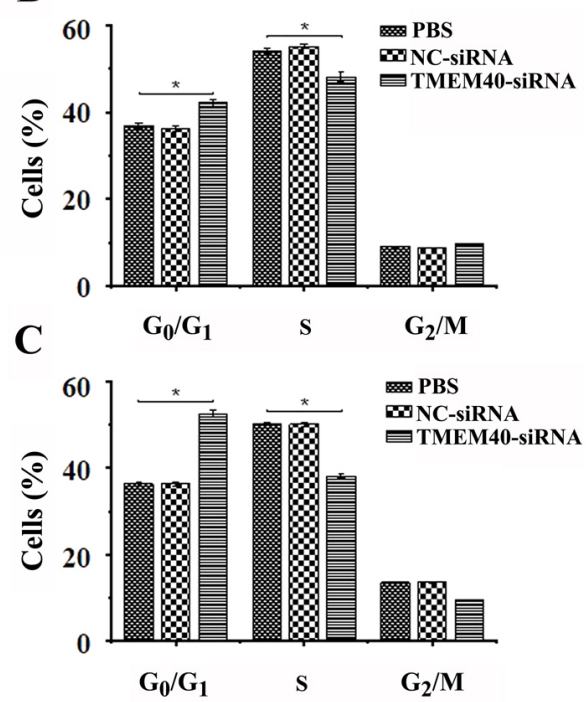

D

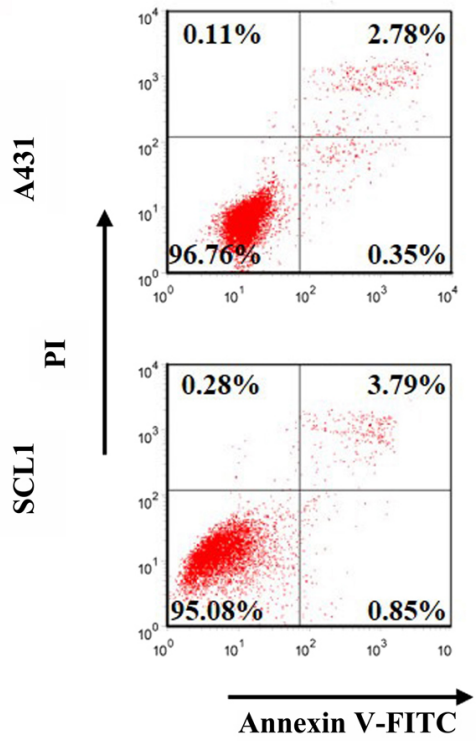

TMEM40-siRNA
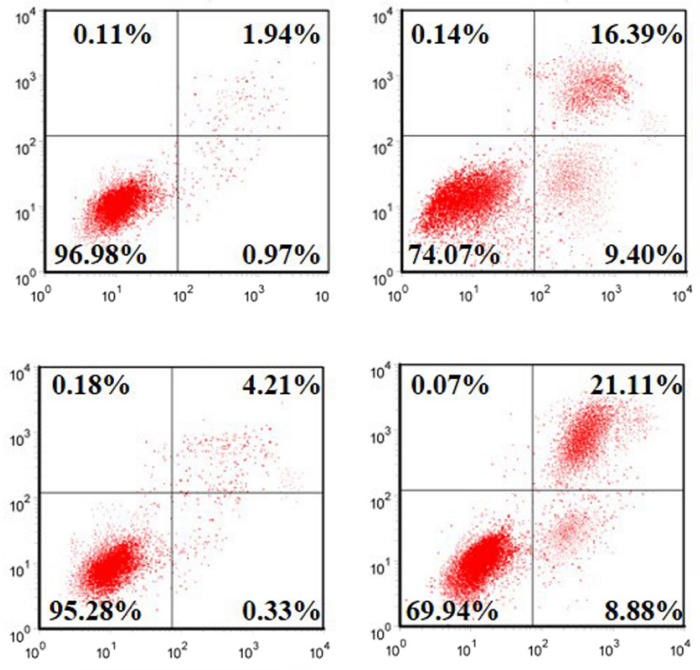

E
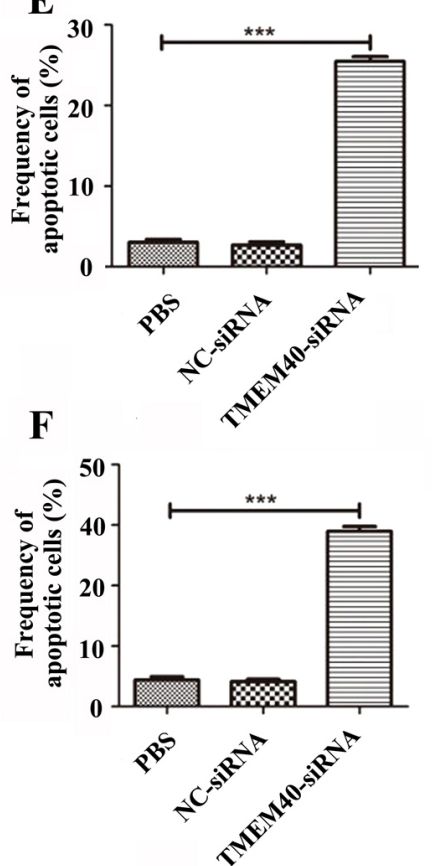

Figure 4. TMEM40 induced cell cycle arrest and apoptosis in A431 and SCL1 cells. (A) Cell cycle distribution in (B) A431 and (C) SCL1 cells following TMEM40-siRNA transfection. (D) Flow cytometry was carried out to evaluate apoptosis in (E) A431 and (F) SCL1 cells transfected with TMEM40-siRNA. ${ }^{*} \mathrm{P}<0.05$, TMEM40-siRNA group vs. NC-siRNA group and PBS group. ${ }^{* * *} \mathrm{P}<0.001$, TMEM40-siRNA group vs. NC-siRNA group and PBS group. CSCC, cutaneous squamous cell carcinoma; NC, negative control; TMEM40, transmembrane protein 40; siRNA, small interfering RNA; PI, propidium iodide; FITC, fluorescein isothiocyanate.

factors of CSCC include ultraviolet radiation exposure, chronic immunosuppressed state, inherited genetic conditions, ionizing radiation exposure, human papillomavirus infection and chronic arsenic exposure (21-25). Moreover, it is necessary to differentiate benign lesions and reactive squamo-proliferative lesions from CSCC $(26,27)$ and to identify the high-risk features associated with invasive tumor progression (28). Therefore, a clear understanding of the molecular mechanism that induces the development and progression of CSCC is essential for the development of diagnostic and prognostic tools, as well as targeted therapies $(29,30)$.

Previous studies have indicated that TMEM40 is upregulated in bladder cancer and tongue squamous cell carcinoma, compared with normal tissue, and that it is involved in cell invasion and migration $(6,10,13,21)$. Therefore, TMEM40 may represent a potential biomarker for new therapeutic strategies and diagnostic development. However, to the best of our knowledge, the molecular mechanism underlying the biological function of TMEM40 in CSCC has not been characterized yet. In the current study, the expression of TMEM40 was evaluated in two CSCC cell lines, in addition to tissue samples, both at the protein and the mRNA levels. TMEM40 gene expression was significantly upregulated in CSCC tissues, compared with adjacent normal skin, and was associated with tumor size, but not with age or sex. Immunohistochemical detection of TMEM40 expression in tissue samples from 
patients with CSCC further supported these results at the protein level. In order to assess the role of TMEM40 on CSCC cell phenotype and to evaluate possible biological functions, TMEM40-siRNA was transfected into A431 and SCL1 cells.

Furthermore, TMEM40 knockdown inhibited the proliferation, migration and invasion of A431 and SCL1 cells. Invasion and migration are the most critical characteristics of malignant tumors. It has been demonstrated that knockdown of TMEM40 expression in clear cell renal cell carcinoma, bladder cancer and tongue squamous cell carcinoma results in significant inhibition of cell proliferation, migration and invasion in vitro $(10,13)$. In the present study, a similar result was also observed, and TMEM40 knockdown resulted in marked reduction in migration and invasion of CSCC cells. These findings provide evidence for the role of TMEM40 in CSCC cell proliferation and motility. Matrix metalloproteinases (MMPs) can promote tumor cell relocation by degrading certain components of the extracellular matrix $(31,32)$. It has been reported that four collagenolytic MMPs, namely MMP-1 and MMP-2, MMP-9 and MMP-13, are associated with CSCC invasion $(32,33)$. It is therefore possible that reduction in MMP production following TMEM40 knockdown impairs implantation of CSCC cells, thus delaying tumor cell growth. Moreover, TMEM40 knockdown may favor the invasion and migration of CSCC cells via multiple downstream MMP-mediated pathways. However, the mechanism underlying this regulatory effect remains to be elucidated in future studies.

TMEM40 silencing inhibited the $\mathrm{G}_{1} / \mathrm{S}$ cell cycle transition and induced apoptosis. Cell cycle analysis following TMEM40 knockdown revealed significant $\mathrm{G}_{0} / \mathrm{G}_{1}$ phase arrest. In addition, downregulation of several genes coding for proteins regulating cell cycle, including cyclin D1, cyclin E, CDK2, CDK4 and CDK6, is associated with tumor progression (34). Cyclin D1 is a key protein in cell cycle regulation that has been shown to be sufficient to drive cell cycle progression (35-37). The present study demonstrated that TMEM40 was involved in the regulation of the cell cycle in CSCC cells and may therefore influence the expression levels of cell cycle proteins.

Furthermore, the role of TMEM40 in the proliferation and apoptosis A431 and SCL1 cells was also evaluated. Functional experiments using CSCC cells revealed that TMEM40 silencing resulted in decreased cell proliferation and increased apoptosis. Apoptosis is a complex process that involves several signaling pathways. Accordingly, the findings of the current study warrant further investigation of TMEM40-related signaling pathways for the clinical treatment of CSCC.

In summary, the present study demonstrated that TMEM40 was significantly upregulated in CSCC cells and tissue, and associated with the pathogenic mechanism of CSCC. Thus, TMEM40 may serve an important role in the development and progression of CSCC. Taken together, the present findings provide insight into the function of TMEM40 in CSCC, which may be used as a potential therapeutic option for CSCC.

\section{Acknowledgements}

Not applicable.

\section{Funding}

No funding was received.

\section{Availability of data and materials}

The datasets used and/or analyzed during the current study are available from the corresponding author on reasonable request.

\section{Authors' contributions}

LY and LLG conceived and designed the study. LY and JL performed the experiments, wrote the manuscript and revised it critically for important intellectual content. XFZ coordinated the research and analyzed the data. WLZ and DLL performed western blot and immunohistochemistry analysis, and were involved in drafting the manuscript. XWQ and TDZ performed the statistical analysis. LLG and TDZ confirm the authenticity of all the raw data. All authors have read and approved the final manuscript.

\section{Ethics approval and consent to participate}

All tissue samples were obtained with written informed consent from the patients involved in this research project. This study was approved by the Medical Ethics Committee of Zhujiang Hospital of Southern Medical University (approval no. 2020-KY-059-01).

\section{Patient consent for publication}

Not applicable.

\section{Competing interests}

The authors declare that they have no competing interests.

\section{References}

1. Kivisaari A and Kähäri VM: Squamous cell carcinoma of the skin: Emerging need for novel biomarkers. World J Clin Oncol 4: 85-90, 2013.

2. Li Y, Huang C and Yang X: Characterization of TCF4-mediated oncogenic role in cutaneous squamous cell carcinoma. Int J Clin Exp Pathol 12: 3583-3594. 2019.

3. Zhang L, Qin H, Wu Z, Chen W and Zhang G: Pathogenic genes related to the progression of actinic keratoses to cutaneous squamous cell carcinoma. Int J Dermatol 57: 1208-1217, 2018.

4. Niu T, Tian Y, Wang G, Guo G, Tong Y and Shi Y: Inhibition of ROS-NF- $\mathrm{kB}$-dependent autophagy enhances Hypocrellin A united LED red light-induced apoptosis in squamous carcinoma A431 cells. Cell Signal 69: 109550, 2020.

5. Burton KA, Ashack KA and Khachemoune A: Cutaneous squamous cell carcinoma: A review of high-risk and metastatic disease. Am J Clin Dermatol 17: 491-508, 2016.

6. Zhang Q, Huang D, Zhang Z, Feng Y, Fu M, Wei M, Zhou J, Huang Y, Liu S and Shi R: High expression of TMEM40 contributes to progressive features of tongue squamous cell carcinoma. Oncol Rep 41: 154-164, 2019.

7. Yue Y, Grossmann B, Ferguson-Smith M, Yang F and Haaf T: Comparative cytogenetics of human chromosome 3 q21.3 reveals a hot spot for ectopic recombination in hominoid evolution. Genomics 85: 36-47, 2005.

8. Yue Y, Grossmann B, Tsend-Ayush E, Grutzner F, FergusonSmith MA, Yang F and Haaf T: Genomic structure and paralogous regions of the inversion breakpoint occurring between human chromosome $3 \mathrm{p} 12.3$ and orangutan chromosome 2. Cytogenet Genome Res 108: 98-105, 2005.

9. Müller S, Stanyon R, Finelli P, Archidiacono N and Wienberg J: Molecular cytogenetic dissection of human chromosomes 3 and 21 evolution. Proc Natl Acad Sci USA 97: 206-211, 2000. 
10. Zhang QY, Fu MT, Zhang ZF, Feng YZ, Wei M, Zhou JY and Shi R: Expression of TMEM40 in bladder cancer and its correlation with clinicopathological parameters. Int J Clin Exp Pathol 10: 8050-8057, 2017.

11. Darai E, Kost-Alimova M, Kiss H, Kansoul H, Klein G and Imreh S: Evolutionarily plastic regions at human 3 p21.3 coincide with tumor breakpoints identified by the 'elimination test'. Genomics 86: 1-12, 2005.

12. $\mathrm{Yu} X$, Teng H, Marques A, Ashgari F and Ibrahim SM: High resolution mapping of Cia3: A common arthritis quantitative trait loci in different species. J Immunol 182: 3016-3023, 2009.

13. Zhang ZF, Zhang HR, Zhang QY, Lai SY, Feng YZ, Zhou Y, Zheng SR, Shi R and Zhou JY: High expression of TMEM40 is associated with the malignant behavior and tumorigenesis in bladder cancer. J Transl Med 16: 9, 2018.

14. Farshchian M, Nissinen L, Siljamäki E, Riihilä P, Piipponen M, Kivisaari A, Kallajoki M, Grénman R, Peltonen J, Peltonen S, et al: Tumor cell-specific AIM2 regulates growth and invasion of cutaneous squamous cell carcinoma. Oncotarget 8 : 45825-45836, 2017.

15. Bottomley MJ, Thomson J, Harwood C and Leigh I: The role of the immune system in cutaneous squamous cell carcinoma Int J Mol Sci 20: 2009, 2019.

16. Lin N, Zhou Y, Lian X and Tu Y: MicroRNA-31 functions as an oncogenic microRNA in cutaneous squamous cell carcinoma cells by targeting RhoTBT1. Oncol Lett 13: 1078-1082, 2017.

17. Stratigos A, Garbe C, Lebbe C, Malvehy J, del Marmol V, Pehamberger H, Peris K, Becker JC, Zalaudek I, Saiag P, et al: Diagnosis and treatment of invasive squamous cell carcinoma of the skin: European consensus-based interdisciplinary guideline. Eur J Cancer 51: 1989-2007, 2015.

18. Farshchian M, Nissinen L, Siljamäki E, Riihilä P, Toriseva M, Kivisaari A, Ala-Aho R, Kallajoki M, Veräjänkorva E, Honkanen HK, et al: EphB2 promotes progression of cutaneous squamous cell carcinoma. J Invest Dermatol 135: 1882-1892, 2015.

19. Livak KJ and Schmittgen TD: Analysis of Relative Gene Expression Data Using Real-Time Quantitative PCR and the 2(-Delta Delta C(T)) method. Methods 25: 402-408, 2001

20. Zhou M, Liu W, Ma S, Cao H, Peng X, Guo L, Zhou X, Zheng L, Guo L, Wan M, et al: A novel onco-miR-365 induces cutaneous squamous cell carcinoma. Carcinogenesis 34 : 1653-1659, 2013.

21. McGuire JF, Ge NN and Dyson S: Nonmelanoma skin cancer of the head and neck I: Histopathology and clinical behavior Am J Otolaryngol 30: 121-133, 2009.

22. Martinez JC and Cook JL: High-risk cutaneous squamous cell carcinoma without palpable lymphadenopathy: Is there a therapeutic role for elective neck dissection? Dermatol Surg 33 410-420, 2007

23. Weinberg AS, Ogle CA and Shim EK: Metastatic cutaneous squamous cell carcinoma: An update. Dermatol Surg 33. 885-899, 2007

24. Li X, Zhou C, Zhang C, Xie X, Zhou Z, Zhou M, Chen L and Ding Z: MicroRNA-664 functions as an oncogene in cutaneous squamous cell carcinomas (cSCC) via suppressing interferon regulatory factor 2. J Dermatol Sci 94: 330-338, 2019.
25. Trakatelli M, Ulrich C, Del MV, Euvrard S, Stockfleth E and Abeni D: Epidemiology of nonmelanoma skin cancer (NMSC) in Europe: Accurate and comparable data are needed for effective public health monitoring and interventions. Br J Dermatol 156 (Suppl 3): S1-S7, 2007.

26. Alam $M$ and Ratner D: Cutaneous squamous-cell carcinoma. N Engl J Med 344: 975-983, 2001.

27. Feng $C$, Zhang HL, Zeng A, Bai $M$ and Wang $X J$ : Tumor-suppressive microRNA-216b binds to TPX2, activating the p53 signaling in human cutaneous squamous cell carcinoma. Mol Ther Nucleic Acids 20: 186-195, 2020.

28. Tian K, Liu W, Zhang J, Fan X, Liu J, Zhao N, Yao C and Miao G: MicroRNA-125b exerts antitumor functions in cutaneous squamous cell carcinoma by targeting the STAT3 pathway. Cell Mol Biol Lett 25: 12, 2020

29. Dlugosz A, Merlino G and Yuspa SH: Progress in cutaneous cancer research. J Investig Dermatol Symp Proc 7: 17-26, 2002.

30. Mavropoulos JC, Aldabagh B and Arron ST: Prospects for personalized targeted therapies for cutaneous squamous cell carcinoma. Semin Cutan Med Surg 33: 72-75, 2014.

31. Liu D, Zhou G, Shi H, Chen B, Sun X and Zhang X: Downregulation of transmembrane protein 40 by miR-138-5p suppresses cell proliferation and mobility in clear cell renal cell carcinoma. Iran J Biotechnol 18: e2270, 2020.

32. Ala-aho R, Ahonen M, George SJ, Heikkilä J, Grénman R, Kallajoki M and Kähäri VM: Targeted inhibition of human collagenase-3 (MMP-13) expression inhibits squamous cell carcinoma growth in vivo. Oncogene 23: 5111-5123, 2004

33. Johansson N, Airola K, Grénman R, Kariniemi AL, Saarialho-Kere U and Kähäri VM: Expression of collagenase-3 (matrix metalloproteinase-13) in squamous cell carcinomas of the head and neck. Am J Pathol 151: 499-508, 1997.

34. Le XF, Bedrosian I, Mao W, Murray M, Lu Z, Keyomarsi K, Lee MH, Zhao J and Bast RC Jr: Anti-HER2 antibody trastuzumab inhibits CDK2-mediated NPAT and histone $\mathrm{H} 4$ expression via the PI3K pathway. Cell Cycle 5: 1654-1661, 2006.

35. Li X, Ding R, Han Z, Ma Z and Wang Y: Targeting of cell cycle and let-7a/STAT3 pathway by niclosamide inhibits proliferation, migration and invasion in oral squamous cell carcinoma cells. Biomed Pharmacother 96: 434-442, 2017.

36. Zhang XQ, Feng H, Li ZY, Guo J and Li M: Aspirin is involved in the cell cycle arrest, apoptosis, cell migration, and invasion of oral squamous cell carcinoma. Int J Mol Sci 19: 2029, 2018.

37. Santamaría D, Barrière C, Cerqueira A, Hunt S, Tardy C, Newton K, Cáceres JF, Dubus P, Malumbres M and Barbacid M: Cdk1 is sufficient to drive the mammalian cell cycle. Nature 448: 811-815, 2007.

This work is licensed under a Creative Commons Attribution-NonCommercial-NoDerivatives 4.0 International (CC BY-NC-ND 4.0) License. 